\title{
Altered Arterial Expression Patterns of Inducible and Endothelial Nitric Oxide Synthase in Pulmonary Plexogenic Arteriopathy Caused by Congenital Heart Disease
}

\author{
ROLF M. F. BERGER, RALF GEIGER, JOHN HESS, AD J. J. C. BOGERS, and WOLTER J. MOOI
}

Departments of Pediatrics, Division of Pediatric Cardiology, Cardiothoracic Surgery and Pathology, Sophia Children's Hospital/University Hospital Rotterdam, The Netherlands; and Department of Pediatric Cardiology, German Heart Center, Technical University of Munich, Munich, Germany

\begin{abstract}
Flow-associated pulmonary hypertension leads to pulmonary plexogenic arteriopathy (PPA), a specific pulmonary vascular disease that includes vascular lesions characterized by abnormal vasodilatation and endothelial cell proliferation. Increased local production of NO has been suggested in this condition. Because reported data on the expression of endothelial NO-synthase (ecNOS) have been contradictory, we speculated that the expression of the inducible isoform of NOS (iNOS) is enhanced in this form of pulmonary hypertension. We investigated immunohistochemically the expression of ecNOS and iNOS in lung tissue of patients with flowassociated pulmonary hypertension $(n=18)$ and compared the findings with those in patients with increased pulmonary blood flow but normal pulmonary artery pressure $(n=10)$, with congestive vasculopathy $(n=6)$ and control subjects $(n=4)$. Immunoreactivity for ecNOS and iNOS was present both in normal and diseased pulmonary arteries. Marked immunoreactivity to both isoforms was present within the advanced lesions of PPA, including plexiform lesions. Semiquantitative analysis of immunoreactivity, both for ecNOS and iNOS, showed no correlation with the severity of morphologic vascular lesions $(p=0.29$ and $p=0.23$, respectively). In contrast to ecNOS, immunoreactivity for iNOS was increased in patients with flow-associated pulmonary hypertension compared with other patients $(p=0.02)$. The present study has demonstrated enhanced expression of iNOS in patients at risk for advanced PPA, but not in patients with other forms of pulmonary arteriopathy. Moreover, high expression of both ecNOS and iNOS were present in advanced lesions of PPA. These data suggest differentiated roles for different isoforms of NOS in the pathogenesis of this specific pulmonary arteriopathy.
\end{abstract}

Pulmonary hypertension is associated with a variety of structural and functional changes of the pulmonary arteries (1-4). These structural changes include medial hypertrophy of the arterial wall and narrowing of the vascular lumen by intimal thickening, resulting in an increase in pulmonary vascular resistance. Pulmonary plexogenic arteriopathy (PPA) is a specific form of pulmonary vascular disease $(1,5)$. PPA may develop in patients with congenital heart disease, where increased pulmonary blood flow appears to be an obligatory factor and elevated pulmonary artery pressure seems to accelerate disease progression $(6,7)$. In contrast to other arteriopathies such as those associated with chronic hypoxia or pulmonary venous congestion, PPA may ultimately cause characteristic vascular alterations, including concentric-laminar intimal fi-

(Received in original form August 31, 1999 and in revised form July 14, 2000)

Dr. Geiger is a recipient of Grant No. J 1282 from the Austrian Science Foundation, Vienna, Austria.

Correspondence and requests for reprints should be addressed to Rolf M. F. Berger, M.D., Ph.D., Department of Pediatrics, Division of Pediatric Cardiology, Sophia Children's Hospital/University Hospital Rotterdam, Dr. Molewaterplein 60, 3015 GJ Rotterdam, The Netherlands. E-mail: berger@alkg.azr.nl

Am J Respir Crit Care Med Vol 163. pp 1493-1499, 2001

Internet address: www.atsjournals.org brosis, dilatation lesions, fibrinoid necrosis of small arteries, and plexiform lesions $(1,3,8)$. Dilatation lesions and plexiform lesions, which are regarded as indicative of irreversibility of the vascular disease, are characterized by local abnormal vasodilatation and endothelial cell proliferation $(5,9)$. The mechanisms through which these characteristic vascular lesions develop are largely unknown. It has become clear that the vascular endothelium, preeminently suited to detect changes in mechanical forces such as cyclic strain and shear stress plays an important role in the pulmonary vascular remodeling process $(10,11)$. Endothelial alterations may cause an imbalance between the production of mediators with vasodilator or vasoconstrictor properties, leading to impaired endothelium-dependent vasorelaxation already early in pulmonary vascular disease, and they may be responsible for increased vascular cell proliferation (12-17). A key role has been attributed to the endothelial-derived relaxing factor nitric oxide (NO), whose pleiotropic effects on blood vessels include vasodilation and stimulation of angiogenesis by migration and proliferation of endothelial cells $(18,19)$. NO is synthesized by the enzyme nitric oxide synthase (NOS) that converts L-arginine to L-citrulline $(18,20-22)$. To date, three isoforms of NOS have been identified. The original classification in "constitutive" endothelial and neuronal isoforms (ecNOS and nNOS, respectively) and an "inducible" isoform (iNOS) is to some extent misleading, because it has become clear that gene expression of both ecNOS and nNOS is upregulated under different conditions (e.g., shear stress, nerve injury) and that, conversely, iNOS is constitutively expressed under physiologic conditions in a variety of cells, including endothelial cells (23). All three isoforms of NOS have been detected in endothelial cells of the normal human pulmonary vasculature $(14,24,25)$. In pulmonary vascular disease, however, published data on the expression of NOS have been contradictory. Giaid and Saleh (14) reported decreased expression of ecNOS in patients with pulmonary hypertension and its absence from plexiform lesions, whereas Mason and coworkers (26) demonstrated high expression of ecNOS in plexiform lesions. In several experimental studies, enhanced expression of ecNOS was found in pulmonary hypertension (27-29). The presence of inducible NOS in pulmonary hypertension has, to our knowledge, not been reported earlier.

Increased NO production may be involved in the abnormal local vasodilation and proliferative lesions, constituting unique features of advanced PPA. Because reported data on the expression of ecNOS in these conditions are contradictory, we hypothesized that the inducible isoform of NOS may be enhanced in flow-associated pulmonary hypertension. In this study we investigated the patterns of expression of ecNOS and iNOS, as evident from immunohistochemistry, in pulmonary arteries of patients with pulmonary plexogenic arteriopathy and related the findings to pulmonary hemodynamics. We fur- 


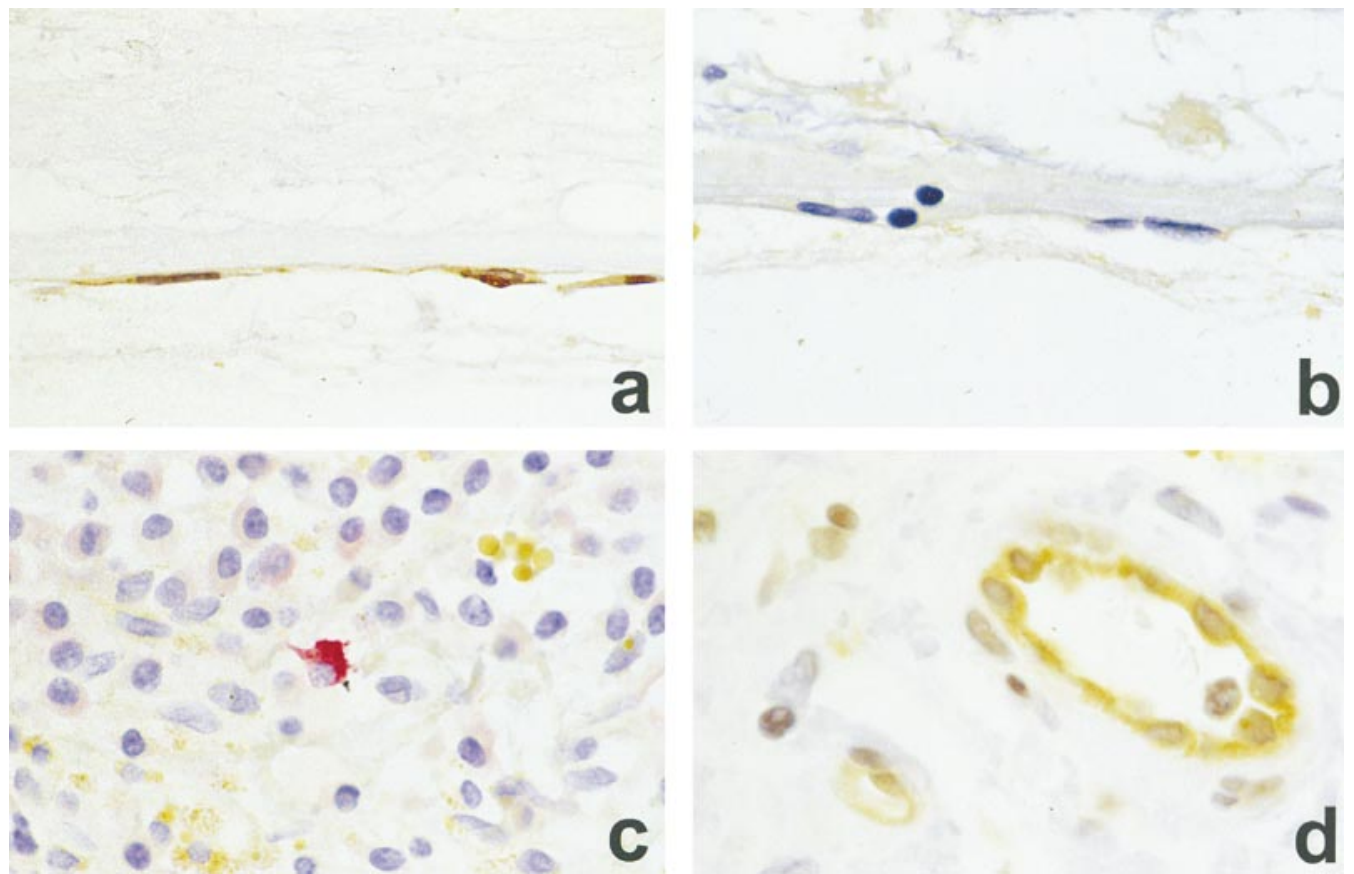

Figure 1. Confirmation of immunoreactivity for ecNOS (immunoperoxidase; brown) and iNOS (alkaline phospatase; red) and specificity of the used antibodies on paraffin material (original magnification: $\times 100)$. (a) Immunostaining in aortic endothelium after incubation with anti-ecNOS antibodies. (b) Negative staining of aortic endothelium after incubation with anti-iNOS antibodies. (c) Immunostaining in activated, synovial macrophages of a patient with rheumatoid arthritis after incubation with anti-iNOS antibodies. (d) Negative staining of synovial macrophages of a patient with rheumatoid arthritis, but immunostaining in the endothelium of synovial vessels after incubation with anti-ecNOS antibodies.

ther compared the findings with those in patients with congestive vasculopathy, where such vasodilation and proliferation does not occur, and in control subjects with a normal pulmonary circulation.

\section{METHODS}

\section{Patients}

Thirty four patients with congenital heart disease who underwent cardiac catheterization and had increased pulmonary blood flow (pulmonary-to-systemic blood flow ratio $>1.2$ ) and/or pulmonary hypertension (mean pulmonary artery pressure $>20 \mathrm{~mm} \mathrm{Hg}$ ) and in whom subsequently a lung tissue specimen was obtained were enrolled in the study, after written informed parental consent. Four patients with a normal pulmonary circulation, who underwent cardiac catheterization and cardiac surgery because of mild left-sided obstructive lesions (pulmonary wedge pressure $<15 \mathrm{~mm} \mathrm{Hg}$ ) served as control subjects. In all patients a complete hemodynamic evaluation was performed, including measurements of pulmonary artery pressure, determination of shunt size by oximetry and calculation of pulmonary and systemic vascular resistance. Blood flow was determined using the dye dilution technique. In addition to the control subjects (Group 1), 10 patients had a congenital heart defect associated with increased pulmonary blood flow and normal pulmonary artery pressure (Group 2), six patients without intracardiac shunts had pulmonary hypertension caused by pulmonary venous congestion (Group 3). Eighteen patients had heart defects associated with both increased pulmonary blood flow and pul-

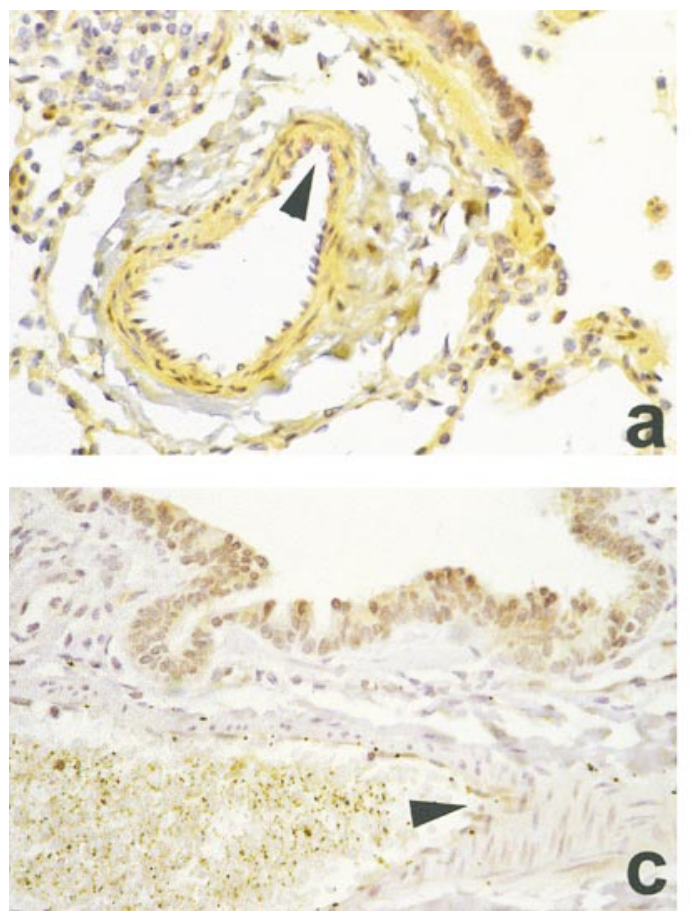

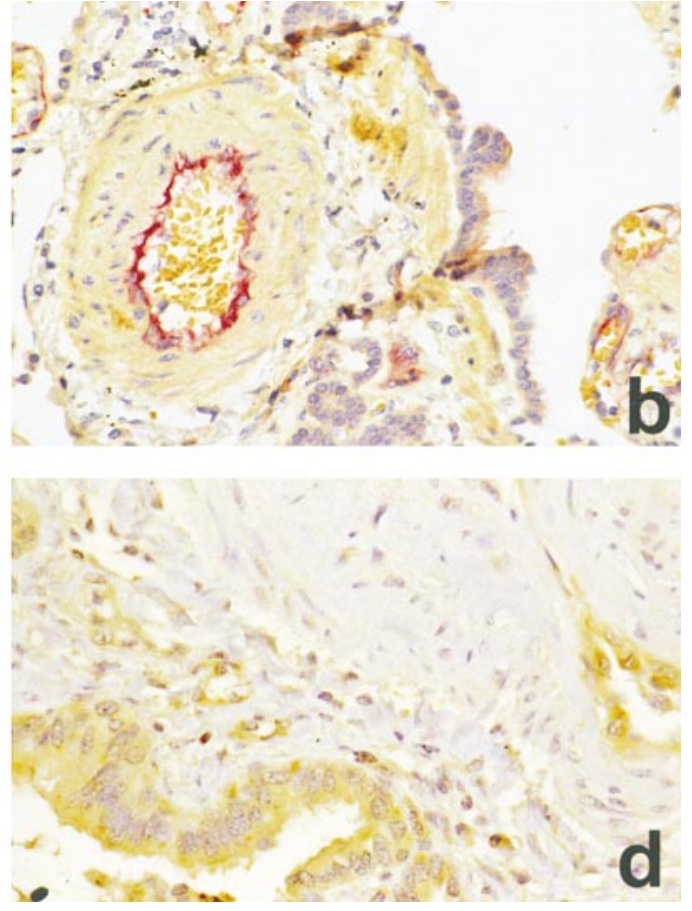

Figure 2. Immunostaining for ecNOS (immunoperoxidase; brown) and iNOS (alkaline phosphatase; red) in lung biopsy sections of patients with various morphologic vascular changes (original magnification: $\times 40)$. (a) Weak immunostaining for iNOS in endothelial cells of a muscular artery in a control patient; there is no specific staining of the bronchiolar epithelium. (b) Pronounced immunostaining for iNOS in the endothelium of an artery with medial hypertrophy in a patient with pulmonary hypertension and increased pulmonary blood flow. (c) Faint immunostaining for ecNOS in endothelial cells of a muscular artery in a control patient; there also is positive staining of the bronchiolar epithelium. (d) Immunostaining for ecNOS in the endothelium of an artery with medial hypertrophy in a patient with pulmonary hypertension and increased pulmonary blood flow. 
TABLE 1. CLINICAL DIAGNOSES OF THE STUDY PATIENTS*

\begin{tabular}{|c|c|c|c|c|c|}
\hline \multicolumn{2}{|l|}{ Diagnosis } & $\begin{array}{c}\text { Group } 1 \\
(n)\end{array}$ & $\begin{array}{c}\text { Group } 2 \\
(n)\end{array}$ & $\begin{array}{c}\text { Group } 3 \\
(n)\end{array}$ & $\begin{array}{c}\text { Group } 4 \\
(n)\end{array}$ \\
\hline \multirow[t]{3}{*}{ VSD } & isolated & & 7 & & 7 \\
\hline & +PDA & & & & 2 \\
\hline & $+\mathrm{ASD}$ & & & & 3 \\
\hline \multicolumn{3}{|l|}{ CAVSD } & & & 4 \\
\hline \multicolumn{3}{|c|}{ PDA } & & & 1 \\
\hline \multicolumn{3}{|c|}{ Left pulmonary artery from aorta } & & & 1 \\
\hline \multicolumn{3}{|c|}{ ASD } & 3 & & \\
\hline \multicolumn{3}{|c|}{ (sub)valv. AoS \pm CoAo $\pm \mathrm{MS}$} & & 6 & \\
\hline \multicolumn{2}{|c|}{ Control subjects } & 4 & & & \\
\hline \multicolumn{2}{|c|}{ Total } & 4 & 10 & 6 & 18 \\
\hline
\end{tabular}

Definition of abbreviations: ASD = atrial septal defect; CAVSD = complete atrioventricular septal defect; $\mathrm{CoAo}=$ coarctation of the aorta; $\mathrm{MS}=$ mitral stenosis; PDA = persistent ductus arteriosus; VSD = ventricular sepal defect.

* Group 1 are control subjects; Group 2 are patients with increased pulmonary blood flow and normal pulmonary artery pressure; Group 3 are patients with pulmonary hypertension caused by chronic pulmonary venous congestion; Group 4 are patients with pulmonary hypertension associated with increased pulmonary blood flow.

monary hypertension (Group 4). The study protocol was approved by the medical ethical committee of the University Hospital Rotterdam.

\section{Tissue Specimens}

Lung tissue was obtained as a biopsy during cardiac surgery $(\mathrm{n}=36)$ or as an open lung biopsy procedure for diagnostic purpose $(n=2)$. Tissue was formalin-fixed under vacuum and paraffin-embedded. Sections $5 \mu \mathrm{m}$ thick were cut at various levels and stained with hematoxylin-eosin (HE) and Elastic-van Gieson (EvG) to allow for proper identification of morphologic structures and precise correlation of morphology with patterns of immunoreactivity. Vascular changes were classified according to Wagenvoort and Wagenvoort (1) and Wagenvoort and Mooi (3).

\section{Immunohistochemistry}

The peroxidase-antiperoxidase (PAP) technique was used for staining ecNOS, and the avidin-biotin complex (ABC) method was used for staining iNOS. Sections of paraffin-embedded lung tissues were deparaffinized, rehydrated in graded ethanols, and rinsed in water. For the PAP method, sections were put in methanol/hydrogen peroxide $3 \%$ for $20 \mathrm{~min}$ to quench endogenous peroxidase. For antigen-retrieval sections were cooked in citric acid buffer for $10 \mathrm{~min}$. Slides were allowed to return to room temperature over several hours to minimize tissue destruction. Subsequently, sections were rinsed in 5\% phosphate-buffered saline (PBS) and incubated with $10 \%$ normal rabbit serum (PAP) or normal goat serum (ABC) for 15 min to block nonspecific binding. The sections were then incubated overnight with mouse monoclonal antibody against either ecNOS in dilution 1:40 or iNOS in dilution 1:50. The antibody reacting with the endothelial NOS was raised against a $140 \mathrm{kD}$ immunogen, corresponding to amino acids 1,030 to 1,209 of the carboxy terminal reductase domain of human ecNOS and has specificity for human, rat and mouse ecNOS (Transduction Laboratories, Lexington, KY). The antibody reacting with the inducible NOS was raised against a $130 \mathrm{kD}$ immunogen of mouse iNOS, corresponding to amino acids 961-1144 of the carboxy terminal half, that has a specificity for human, dog, rat and mouse iNOS (Transduction Laboratories). Both antibodies are monospecific for their NOS isoform and previously showed no cross-reactivity using Western blotting techniques. Reactivity on paraffin material was confirmed by using aortic endothelium for ecNOS and activated macrophages in rheumatoid arthritis for iNOS as positive controls (Figure 1). Incubation with anti-iNOS resulted in negative staining of aortic endothelium. Incubation with anti-ecNOS resulted in negative staining of synovial macrophages but positive staining of endothelial cells in synovial vessels (Figure 1). After rinsing once with PBS-tween $0.2 \%$, and once with PBS 5\% the sections were incubated for $30 \mathrm{~min}$ with rabbit antimouse serum in a dilution of 1:25 (DAKO, Glostrup, Denmark), rinsed with PBS 5\% and incubated with mouse PAP-complex 1:300 (Sigma Chemical CO, St. Louis, MO) for another $30 \mathrm{~min}$. Chromogen consisted of 3,3'-diaminobenzidine tetrahydrochloride (DAB)/hydrogen peroxide in PBS 5\%. The chromogen reaction was allowed to take place over $7 \mathrm{~min}$ in the dark. Sections were rinsed with water, lightly counterstained with Mayer's hematoxylin, and dehydrated in graded alcohols to xylene. For iNOS detection, sections were incubated with biotinylated anti-immunoglobulins (Multilink; Biogenex, San Ramon, $\mathrm{CA}$ ) in dilution $1: 50$ with $2 \%$ normal goat serum and $2 \%$ normal human serum for $30 \mathrm{~min}$, followed by incubation with preformed avidinbiotin-alkaline phosphatase complex (ABC; Biogenex) 1:50 for another $30 \mathrm{~min}$. Chromogen consisted of naphtol AS-MX-phosphate, new fuchsin, sodium nitrite, and levamisole in TRIS- $\mathrm{HCl}$ at $\mathrm{pH} 8.0$, which was added for $30 \mathrm{~min}$. Sections were lightly counterstained with Mayer's hematoxylin and mounted in an aqueous-base mounting medium. Negative controls consisted of replacement of the primary antibody by nonimmunogen serum (DAKO). Finally, serial sections were incubated with monoclonal antibody against CD31, as an endothelial celltype marker, and with a monoclonal antibody against smooth muscle $\alpha$-actin (both DAKO) using routine techniques.

\section{Immunohistochemical Analysis}

All muscular arteries present at the tissue sections were studied. Pulmonary arteries were categorized in preacinar arteries, accompanied by a bronchus or bronchiolus and in intraacinar arteries, not accompanied by a bronchus or bronchiolus. The intensity of the immunostaining in the arteries was graded semiquantitatively from 0 to 3 , with 0 corresponding to the absence of staining, 1 corresponding to the weak staining as identified in controls, 2 to moderate staining, and 3 representing strong staining. The grading was performed by two of the investigators, who were unaware of the clinical and hemodynamic data.

\section{Statistical Analysis}

Interobserver agreement for grades of immunoreactivity for ecNOS and iNOS was determined by calculating intraclass correlation coeffi-

TABLE 2. CHARACTERISTICS OF THE STUDY PATIENTS*

\begin{tabular}{|c|c|c|c|c|}
\hline & $\begin{array}{c}\text { Group 1 } \\
\text { Normal Qp } \\
\text { Normal Ppa } \\
\quad(n=4)\end{array}$ & $\begin{array}{c}\text { Group } 2 \\
\text { Increased Q } \mathrm{p} \\
\text { Normal Ppa } \\
(n=10)\end{array}$ & $\begin{array}{c}\text { Group 3 } \\
\text { Normal Q p } \\
\text { Increased Ppa } \\
(n=6)\end{array}$ & $\begin{array}{c}\text { Group 4 } \\
\text { Increased Qp } \\
\text { Increased Ppa } \\
(n=18)\end{array}$ \\
\hline \multicolumn{5}{|l|}{ Age } \\
\hline Median, yr & 3.3 & 6.6 & 2.4 & 0.7 \\
\hline Range & $1.8-4.8$ & $0.6-32.1$ & $1.1-10.9$ & $0.3-30.7$ \\
\hline \multicolumn{5}{|l|}{ Hemodynamincs } \\
\hline$\overline{\mathrm{Ppa}}, \mathrm{mm} \mathrm{Hg}$ & $13 \pm 2$ & $13 \pm 4$ & $31 \pm 12$ & $41 \pm 14$ \\
\hline Ppcu, mm Hg & $9 \pm 2$ & $7 \pm 2$ & $16 \pm 4$ & $10 \pm 2$ \\
\hline Shunt size,(Q்p/Q்s) & $1.0 \pm 0$ & $2.3 \pm 1.8$ & $1.0 \pm 0$ & $3.1 \pm 1.5$ \\
\hline PVR, $\left(\mathrm{WU} \cdot \mathrm{m}^{2}\right)$ & $0.9 \pm 0.4$ & $1.4 \pm 0.8$ & $5.0 \pm 4.0$ & $5.1 \pm 3.8$ \\
\hline
\end{tabular}

Definition of abbreviations: $\mathrm{Ppa}=$ pulmonary artery pressure; Increased $\mathrm{Ppa}=\overline{\mathrm{Ppa}}>20 \mathrm{~mm} \mathrm{Hg}$; Ppcw $=$ pulmonary capillary wedge pressure; PVR = pulmonary vascular resistance; $\dot{\mathrm{Q} p}=$ pulmonary blood flow; Increased $\dot{\mathrm{Q} p}=\dot{\mathrm{Q} p} / \dot{\mathrm{Q}} \mathrm{s}>1.2 ; \dot{\mathrm{Q}} \mathrm{s}=$ systemic blood flow; WU, Wood's units.

* For definition of groups, see Table 1. 
cients, and these were 0.96 and 0.93 , respectively. No differences of more than one grade between the two investigators was observed. For further analyses the mean of both scores was used. For comparison of continuous variables between groups analysis of variance (ANOVA) or the Kruskal-Wallis tests were used, when appropriate. For comparison of categorical variables between groups chi-square tests were used. Correlation coefficients were calculated using the linear regression technique. A p value $<0.05$ was considered significant. Values are presented as means \pm SD unless indicated otherwise.

\section{RESULTS}

There were 38 patients enrolled in the study. Anatomic diagnoses of the congenital heart disease and the clinical and hemodynamic characteristics of the patients are listed in Tables 1 and 2. Lung tissue specimens stained with HE and EvG revealed essentially normal pulmonary arteries in the control group, and the complete spectrum of morphologic vascular changes, ranging from muscular hypertrophy and intima hyperplasia to the characteristic lesions of advanced plexogenic arteriopathy were found in the patients. Medial thickness was $9 \pm 3 \%$ in the control group (mean \pm SD) and $7 \pm 3,14 \pm 4$, and $16 \pm 4 \%$ in the patient Groups 2, 3, and 4, respectively. Of the patients with increased pulmonary blood flow and normal pulmonary artery pressure (Group 2) two patients showed intimal proliferation as most advanced vascular lesion, whereas in the patients with pulmonary hypertension but normal pulmonary flow (Group 3), one patient showed intimal proliferation as most advanced lesion. In these groups no patients showed concentric laminar intimal fibrosis, dilatation lesions, or plexiform lesions in their lung biopsy. Of the patients with both increased pulmonary blood flow and pulmonary hypertension (Group 4), three showed intimal proliferation as most advanced lesion, one showed concentric laminar intimal fibrosis as most advanced lesion, and six showed dilatation lesions and/or plexiform lesions as most advanced lesions.

\section{Endothelial Nitric Oxide Synthase}

In the control group, immunohistochemical analysis revealed faint staining for ecNOS in pulmonary vascular endothelial cells and also in bronchiolar epithelium, (Figure 2c). Also in the patients a positive staining of vascular endothelium was present in muscular arteries, both with or without morphologic changes (Figure 2d). No differences in staining pattern were observed between preacinar and intraacinar muscularized arteries. Within the advanced lesions of plexogenic arteriopathy positive immunoreactivity was observed. In concentric laminar intimal fibrosis, positivity was seen in endothelial cells but not in the subintimal cells (Figure 3a). In plexiform lesions, strong immunostaining for ecNOS was present in several of the cells forming the plexus (Figure 3c). Immunoreactivity was not found in the cells lining dilated angiomatoid vessels. In 25 of the 34 patients, the intensity of the immunoreaction in the endothelium of muscular arteries, graded semiquantitatively, was comparable to the control subjects. In nine patients, the level of immunostaining of the vascular endothelium was increased in muscular arteries of all sizes. However, semiquantitative grading of the immunoreaction in muscular arteries did not correlate with the severity of the morphologic changes $(p=0.29)$ (Figure 4$)$. Seven of the nine patients with increased intensity of staining were patients with pulmonary hypertension and increased flow (Group 4). Differences between patient groups in endothelial staining intensity did not reach statistical significance $(\mathrm{p}=0.18)$ (Figure 5). However, patients with an increased level of immunoreactivity had a larger pulmonary-to-systemic blood flow ratio $(3.2 \pm 1.3$ versus $2.0 \pm 1.6 ; \mathrm{p}=0.019)$ and a higher pulmonary pulse pres- sure $(40 \pm 13$ versus $25 \pm 13 \mathrm{~mm} \mathrm{Hg} ; \mathrm{p}=0.008)$ than did other patients. No differences in mean pulmonary artery pressure or pulmonary vascular resistance could be demonstrated between patients with different staining levels.

\section{Inducible Nitric Oxide Synthase}

In control subjects, immunohistochemical analysis revealed weak staining for iNOS in pulmonary vascular endothelial cells, but not in bronchiolar epithelium (Figure 2a). Positive staining for iNOS was present in all patients. However, the intensity of the immunoreaction differed remarkably between patients (Figure 2b). No differences in staining pattern were observed between muscular arteries that were accompanied by a bronchus or bronchiole and intraacinar arteries. Intense immunostaining was present in the endothelium of arteries affected by concentric laminar intimal fibrosis, as long as the vessel was not completely occluded (Figure 3b). Also in the cellular plexus of plexiform lesions an increased immunoreaction for iNOS was present (Figure 3d). In addition, endothelial cells lining dilated and angiomatoid vessels showed iNOS positivity. Semiquantitative analysis of the immunoreactivity in muscular arteries revealed no significant correlation with the severity of morphologic vascular changes $(p=0.23)$ (Figure 4). The immunoreactivity to iNOS in these arteries differed significantly between the patient groups $(\mathrm{p}=0.02)$ (Figure 5). The level of immunostaining in patients with both increased pulmonary blood flow and pulmonary hypertension was higher than in control subjects $(\mathrm{p}=0.014)$ and in patients with increased pulmonary blood flow and normal pulmonary artery pressure $(p=0.016)$. The level of immunoreactivity showed a weak, positive correlation with the size of the left-toright shunt $(\mathrm{r}=0.39 ; \mathrm{p}=0.019)$ (Figure 6) and the pulse pressure $(\mathrm{r}=0.35 ; \mathrm{p}=0.034)$, but not with mean pulmonary artery pressure or pulmonary vascular resistance.

\section{DISCUSSION}

The present study has demonstrated that both the endothelial and inducible isoforms of nitric oxide synthase are expressed in the endothelium of muscular arteries in the lungs of children with a normal pulmonary circulation, as well as in those of patients with congenital heart disease associated with pulmonary hypertension and/or increased pulmonary blood flow. The level of immunoreactivity for iNOS in muscular pulmonary arteries was significantly increased in patients with both pulmonary hypertension and increased pulmonary blood flow, which are the patients at highest risk for irreversible PPA. No correlation was found between iNOS and ecNOS expression and the severity of the histologic vascular changes. However, within the characteristic vascular lesions of advanced plexogenic arteriopathy, including concentric laminar intimal fibrosis, dilatation lesions, and plexiform lesions, marked immunoreactivity for both ecNOS and iNOS was observed.

The process of pulmonary vascular remodeling is associated with an impaired endothelial-dependent vasorelaxation, already early in its course $(17,30)$. As such, the finding of increased expression of NOS protein in patients with pulmonary hypertension appears to be paradoxical. However, it has to be acknowledged that increased NOS expression is not synonymous with increased NO production or activity. To date, it remains uncertain whether impaired endothelium-dependent vasorelaxation in flow-related pulmonary hypertension is associated with decreased NO activity. Also other alterations in endothelial cell metabolism that has been suggested to occur in pulmonary hypertension, may be primarily responsible for the impaired endothelial vasorelaxation, including increased 

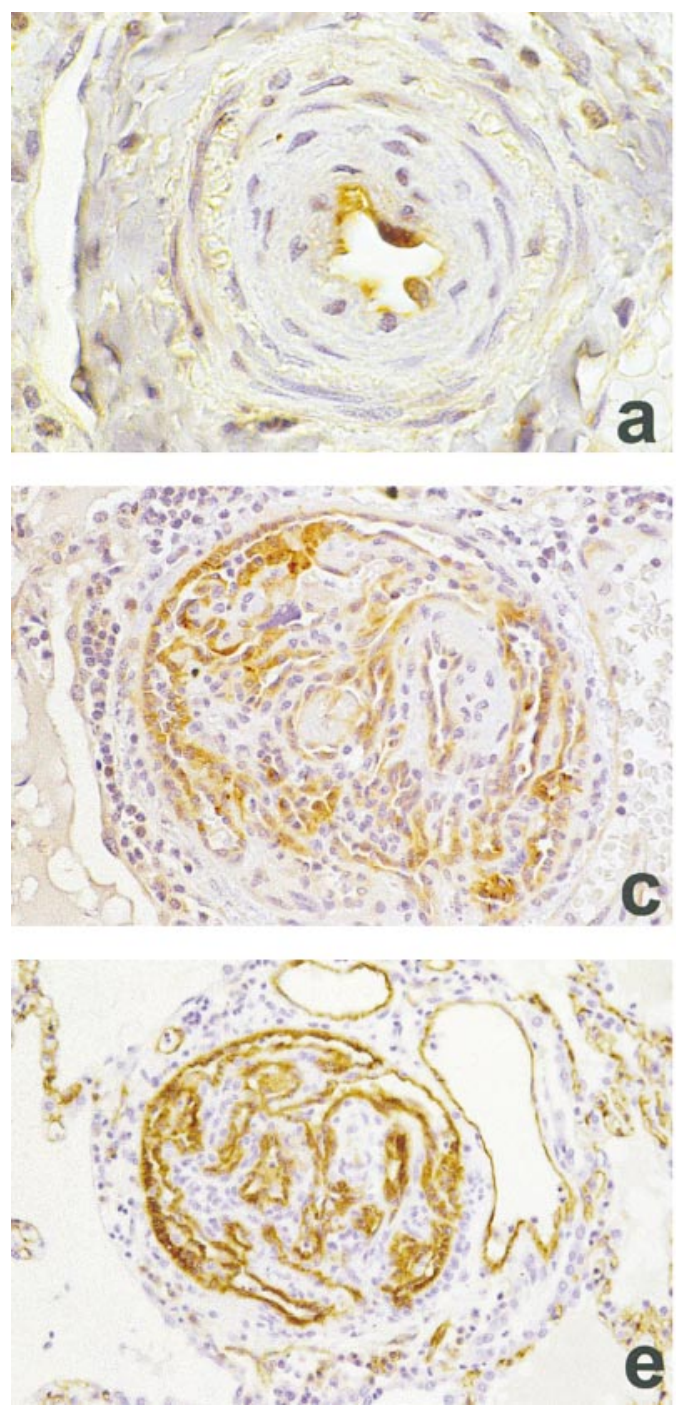
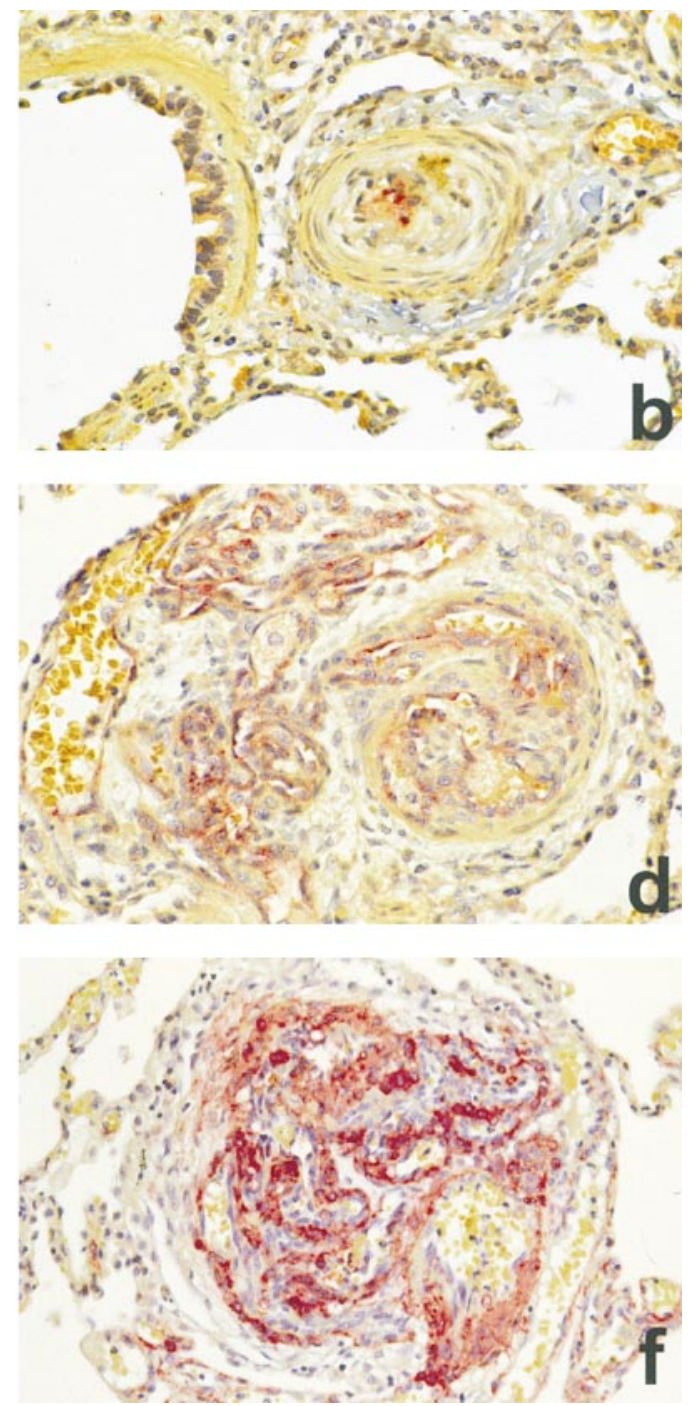

Figure 3. Immunostaining for ecNOS and CD31(immunoperoxidase; brown) and iNOS and $\alpha$-smooth muscle antigen (alkaline phospatase; red) in lung biopsy sections of patients with various morphologic vascular changes. (a) Immunostaining for ecNOS in endothelial cells of a pulmonary artery affected by severe concentric laminar intimal fibrosis (original magnification: $\times 100)$. (b) immunostaining for iNOS in endothelial cells of a pulmonary artery affected by severe concentric laminar intimal fibrosis (original magnification: $\times 40$ ). (c) Distinct immunostaining for ecNOS in cells within a plexiform lesion (original magnification: $\times 40)$. (d) distinct immunostaining for iNOS in cells within a plexiform lesion (original magnification: $\times 40$ ). (e) Distinct immunostaining for CD31 in cells within a plexiform lesion (original magnification: $\times 40)$. ( $f$ ) Distinct immunostaining for $\alpha$-smooth muscle antigen in cells within a plexiform lesion (original magnification: $\times 40$ ). Note that endothelial cells, as characterized by CD31 positivity, are localized adjacent to the lumen of of the slitlike channels in the plexus, whereas the $\alpha$-SMA positive cells, myofibroblasts, are localized in the nonadluminal cells of the plexus. Expression of ecNOS and iNOS shows colocalization with the CD31 positive cells. production of vasoconstrictive mediators or decreased production of prostacyclin or a combination of these $(13,15,16)$. In other words, impaired endothelial-dependent vasorelaxation may occur despite increased NO activity. As mentioned before, published data on the expression of ecNOS in PPA has been conflicting. In contrast to the findings of Giaid and Saleh (14), our data indicate that the expression of ecNOS protein was not decreased in pulmonary vascular endothelium of children with congenital heart disease and did not correlate with the severity of morphologic vascular lesions. Interestingly, the expression of iNOS in pulmonary endothelial cells, which has to our knowledge not been studied previously in patients with pulmonary hypertension, was significantly increased in flowassociated pulmonary hypertension. Although the observed associations between NOS expression and pulmonary hemodynamics obviously do not constitute conclusive evidence for a causal relation, it is in concordance with experimental data that pulmonary NOS expression is upregulated by shear stress and cyclic strain $(18,29,31)$. This is further supported by the identification of a "shear stress-responsive element" in both the human ecNOS and iNOS gene, similar to that found in the promotor of several shear stress inducible endothelial genes (32). Moreover, in pulmonary hypertension associated with congenital heart disease, it has been recognized clinically for years that the characteristic vascular lesions of PPA, including ab- normal, local vasodilatation, and endothelial cell proliferation, occur only in those cases, in which pulmonary hypertension is associated with increased pulmonary blood flow $(1-3,5)$. This is confirmed by the histologic findings in the present study.

In the light of these observations it is tempting to speculate that, in flow-associated pulmonary hypertension, increased amounts of $\mathrm{NO}$ are produced as an adaptive response of the pulmonary circulation aiming to lower pulmonary vascular resistance in response to increased pulmonary blood flow and pressure. The constitutive forms of NOS synthesize small amounts of NO on demand. Stimuli, including shear stress and cyclic strain, lead to an increase in cytosolic calcium, which activates NOS to give off a brief burst of NO (18). In contrast, iNOS is functionally calcium-independent and releases large amounts of NO continuously (18). The enhanced expression of iNOS in the course of flow-associated pulmonary hypertension may explain the evidence for increased production of NO that has been found in both clinical and experimental studies in these conditions $(33,34)$.

Despite increased NO production in flow-associated pulmonary hypertension, NO-mediated vasodilation may not be apparent in the parabronchial and parabronchiolar (axial) pulmonary arteries, where a thickening of the muscular media and fibrous adventitia are almost invariably present, and where other factors such as enhanced vasoconstrictive activity 


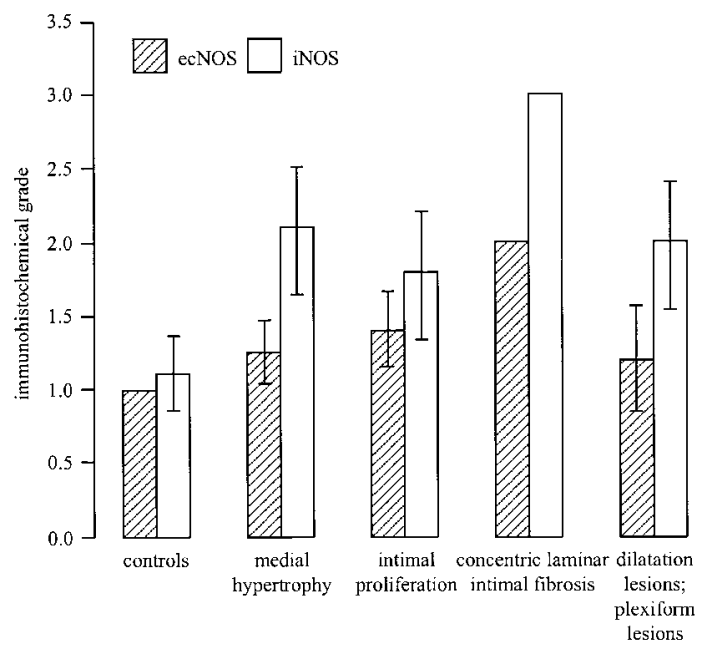

Figure 4. Relation between the semiquantative grading of immunoreactivity to the endothelial, respectively, inducible isoform of nitric oxide synthase in muscular arteries and the morphologic vascular changes. No significant differences in immunoreactivity between the various vascular changes were demonstrated. Data are expressed as mean \pm SD.

may be responsible for a net vasoconstrictive effect. However, from these larger and relatively thick-walled arteries, small branches arise and almost directly enter the alveolated parenchyma. At the origin of these delicate, so-called supernumerary branches, vessel wall damage appears to be greatest, which may relate to high mechanical stress at this particular site (1, 3). Dilatation lesions and plexiform lesions are especially prominent and numerous in these supernumerary arteries (35). From our data, it would appear that the abnormal, local dilatation and cellular proliferation at this site, characteristic of advanced PPA, may be related to increased local levels of NO. Within these vascular lesions we consistently demonstrated marked expression of both ecNOS and iNOS. The finding that immunoreactivity against both ecNOS and iNOS was present in pulmonary arteries with severe concentric laminar intimal fibrosis, until the vessel was completely occluded,

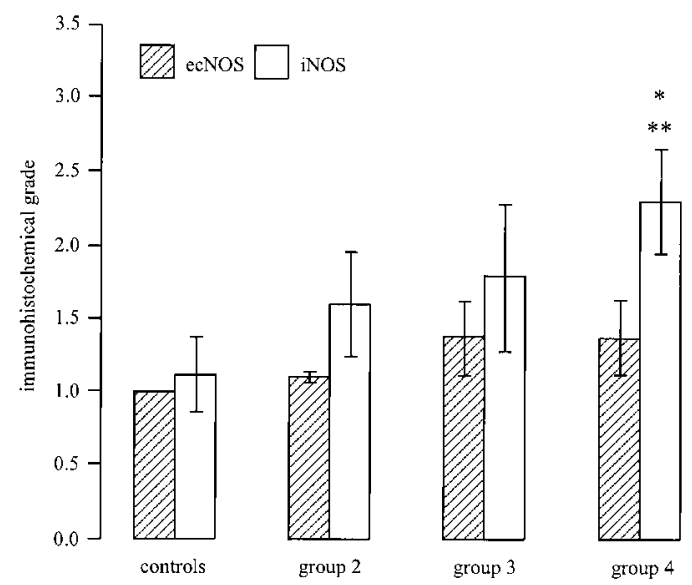

Figure 5. The immunoreactivity to endothelial and inducible nitric oxide synthase, as graded semiquantitatively, in the different patient groups. Patients with pulmonary hypertension, associated with increased pulmonary blood flow (Group 4) revealed significantly higher immunoreactivity than did control subjects and patients with increased pulmonary blood flow but normal pulmonary artery pressure (Group 2); * indicates $p<0.05$ compared with control subjects; ${ }^{* *} p<$ 0.05 compared with Group 2. Data are expressed as mean \pm SD.

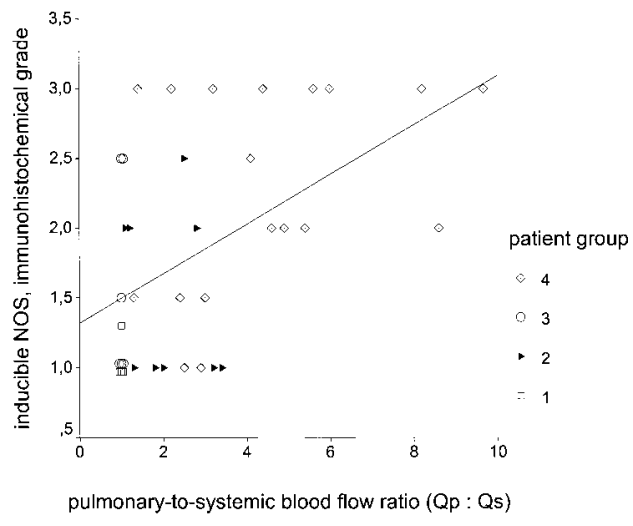

Figure 6. Relationship between the ratio of pulmonary ( $\dot{Q} p)$ to systemic (Qs) blood flow, reflecting a measure for increased pulmonary blood flow, and the grade of immunoreactivity for inducible nitric oxide synthase (iNOS) for the total patient group $(r=0.39, p=0.02)$.

suggests prolonged local NO production until late in plexogenic arteriopathy. The large amounts of NO, that are characteristically produced by iNOS, may attribute to oxidative wall injury and may relate to the development of the vascular lesions of advanced PPA $(18,25)$. Although no cause-effect relation can be deduced from this study, our results provide evidence in support of the view that flow-associated pulmonary hypertension may result in largely increased NO production and local, NO-mediated dilatatory and proliferative effects.

\section{Limitations of the Study}

Immunohistochemistry can demonstrate the presence of a protein but not its activity. An advantage of the immunohistochemistry technique, important to this study, is that it demonstrates directly the microanatomical localization of the protein under study. Our data do not allow conclusions on a possible causal relationship between the parameters assessed in our study. In addition, semiquantitative analysis of immunohistochemical data cannot be more than an estimation of relative differences in staining intensity, and it may be hampered by confounding factors. We have rigorously minimized the possible influence of these factors by rigid standardization of the processing of biopsy material, and by staining of the entire series of sections simultaneously and by relating staining intensity to a standard staining. Persistence of immunoreactivity does not preclude a certain decrease in protein content. On the other hand, however, increased immunoreactivity, as demonstrated for iNOS, can only be explained by an increased presence of the protein under study. Although quantification of this increase by Western blots or ELISA analysis could strengthen the immunohistochemical findings, the amounts of human lung tissue obtained by lung biopsy, did not allow the application of these techniques.

In conclusion, this descriptive study has demonstrated characteristic patterns of the expression of ecNOS and iNOS in the pulmonary vascular remodeling process in human pulmonary hypertension. The increased presence of iNOS in the pulmonary vascular endothelium of patients at high risk for advanced PPA and the marked expression of both isoforms of NOS in dilatation lesions and plexiform lesions may be relevant to the pathogenis of these characteristic vascular lesions of advanced PPA.

\section{References}

1. Wagenvoort CA, Wagenvoort N. Pathology of Pulmonary Hypertension. New York: John Wiley and Sons Inc.; 1977. 
2. Hoffman JI, Rudolph AM, Heymann MA. Pulmonary vascular disease with congenital heart lesions: pathologic features and causes. Circulation 1981;64:873-877.

3. Wagenvoort CA, Mooi WJ. Biopsy pathology of the pulmonary vasculature: biopsy pathology. London: Chapman and Hall Medical; 1989.

4. Haworth SG. Pathophysiological and metabolic manifestations of pulmonary vascular disease in children. Herz 1992;17:254-261.

5. Wagenvoort CA. Open lung biopsies in congenital heart disease for evaluation of pulmonary vascular disease: predictive value with regard to corrective operability. Histopathology 1985;9:417-436.

6. Ivy DD, Neish SR, Abman SH. Regulation of the pulmonary circulation. In: Garson Jr. A, Bricker JT, Fisher DJ, Neish SR, editors. The Science and Practice of Pediatric Cardiology. Vol. 1. Baltimore: William and Wilkins. 1998. p. 329-347.

7. Botney MD. Role of hemodynamics in pulmonary vascular remodeling. Am J Respir Crit Care Med 1999;159:361-364.

8. Heath D, Edwards J. The pathology of hypertensive pulmonary vascular disease: a description of six grades of structural changes in the pulmonary arteries with special reference to congenital cardiac septal defects. Circulation 1958;18:533-547.

9. Tuder RM, Groves B, Badesch DB, Voelkel NF. Exuberant endothelial cell growth and elements of inflammation are present in plexiform lesions of pulmonary hypertension. Am J Pathol 1994;144:275-285.

10. Davies PF. Flow-mediated endothelial mechanotransduction. Physiol Rev 1995;75:519-560.

11. Furchgott RF, Zawadzki JV. The obligatory role of endothelial cells in in the relaxation of arterial smooth muscle by acetylcholine. Nature 1980;288:373-376.

12. Stewart DJ, Levy RD, Cernacek P, Langleben P. Increased plasma endothelin-1 in pulmonary hypertension: marker or mediator of disease? Ann Intern Med 1991;114:464-469.

13. Giaid A, Yanagisawa M, Langleben D, Michel RP, Levy R, Shennib H, Kimura S, Masaki T, Duguid WP, Stewart DJ. Expression of endothelin-1 in the lungs of patients with pulmonary hypertension. $N$ Engl J Med 1993;328:1732-1739.

14. Giaid A, Saleh D. Reduced expression of endothelial nitric oxide synthase in the lungs of patients with pulmonary hypertension. $N$ Engl J Med 1995;333:214-221.

15. Christman BW, McPherson CD, Newman JH, King GA, Bernard GR, Groves BM, Loyd JE. An imbalance between the excretion of thromboxane and prostacyclin metabolites in pulmonary hypertension. $N$ Engl J Med 1992;327:70-75.

16. Tuder RM, Cool CD, Geraci MW, Wang J, Abman SH, Wright L, Badesch D, Voelkel NF. Prostacyclin synthase expression is decreased in lungs from patients with severe pulmonary hypertension. Am J Respir Crit Care Med 1999;159:1925-1932.

17. Celermajer DS, Cullen S, Deanfield JE. Impairment of endotheliumdependent pulmonary artery relaxation in children with congenital heart disease and abnormal pulmonary hemodynamics. Circulation 1993;87:440-446

18. Angard E. Nitric oxide: mediator, murderer, and medicine. Lancet 1994; 343:1199-1206.

19. Fukuo K, Inoue T, Morimoto S, Nakahashi T, Yasuda O, Kitano S, Sas- ada R, Ogihara T. Nitric oxide mediates cytotoxicity and basic fibroblast growth factor release in cultured vascular smooth muscle cells: a possible mechanism of neovascularization in atherosclerotic plaques. J Clin Invest 1995;95:669-676.

20. Celermajer DS, Dollery C, Burch M, Deanfield JE. Role of endothelium in the maintenance of low pulmonary vascular tone in normal children. Circulation 1994;89:2041-2044.

21. Stamler JS, Loh E, Roddy MA, Currie KE, Creager MA. NO regulates basal systemic and pulmonary vascular resistance in healthy humans. Circulation 1994;89:2035-2040.

22. Peacock AJ, Dawes KE, Laurent GJ. Endothelial cell derived growth factors in pulmonary vascular hypertension. Eur Respir Rev 1993;3: 638-643.

23. Michel T, Feron O. Nitric oxide synthases: which, where, how and why? J Clin Invest 1997;100:2146-2152.

24. Kobzik L, Bredt DS, Lowenstein CJ, Drazen J, Gaston B, Sugarbaker D, Stamler JS. Nitric oxide synthase in human and rat lung: immunocytochemical and histochemical localization. Am J Respir Cell Mol Biol 1993;9:371-377.

25. Nathan C. Inducible nitric oxide synthase: what difference does it make? J Clin Invest 1997;100:2417-2423.

26. Mason NA, Springall DR, Burke M, Pollock J, Mikhail G, Yacoub MH, Polak JM. High expression of endothelial nitric oxide synthase in plexiform lesions of pulmonary hypertension. J Pathol 1998;185:313-318.

27. Xue C, Johns RA. Upregulation of nitric oxide synthase correlates temporally with onset of pulmonary vascular remodeling in the hypoxic rat. Hypertension 1996;28:743-753.

28. Resta TC, Gonzales RJ, Dail WG, Sanders TC, Walker BR. Selective upregulation of arterial endothelial nitric oxide synthase in pulmonary hypertension. Am J Physiol 1997;272:H806-H813.

29. Black SM, Fineman JR, Steinhorn RH, Bristow J, Soifer SJ. Increased endothelial NOS in lambs with increased pulmonary blood flow and pulmonary hypertension. Am J Physiol 1998;275:H1643-H1651.

30. Dinh Xuan AT, Higenbottam TW, Clelland C, Pepke-Zaba J, Cremona $\mathrm{G}$, Wallwork J. Impairment of pulmonary endothelium-dependent relaxation in patients with Eisenmenger's syndrome. $\mathrm{Br} J$ Pharmacol 1989;99:9-10

31. Nadaud S, Philippe M, Arnal JF, Michel JB, Soubrier F. Sustained increase in aortic endothelial nitric oxide synthase expression in vivo in a model of chronic high blood flow. Circ Res 1997;79:857-863.

32. Shaul PW. Ontogeny of nitric oxide in the pulmonary vasculature. Semin Perinatol 1997;21:381-392.

33. Takaya J, Teraguchi M, Nogi S, Ikemoto Y, Kobayashi Y. Relation between plasma nitrate and mean pulmonary arterial pressure in ventricular septal defect. Arch Dis Child 1998;79:498-501.

34. Reddy VM, Wong J, Liddicoat JR, Johengen M, Chang R, Fineman JR. Altered endothelium-dependent responses in lambs with pulmonary hypertension and increased pulmonary blood flow. Am J Physiol 1996;271:H562-H570.

35. Yaginuma G, Mohri H, Takahashi T. Distribution of arterial lesions and collateral pathways in the pulmonary hypertension of congenital heart disease: a computer aided reconstructive study. Thorax 1990;45:586-590. 